P79 (continued)

may be an important factor in participant dietary quality, the evaluation of which may be used to inform nutrition education and other interventions directed to the low-income population. The objective of this study was to determine whether food group intake varies by food security status in a sample of Indiana SNAP-Ed eligible adults.

Study Design, Setting, Participants: SNAP-Ed eligible adults age $\geq 18$ y $(n=261)$ from 31 Indiana counties were recruited to participate in this cross-sectional study from August 2015 to May 2016.

Outcome Measures and Analysis: Baseline household adult food security score was assigned using the 10-item United States Household Food Security Survey Module and classified participants as food secure (0-2) or food insecure (3-10). Baseline Healthy Eating Index-2010 (HEI2010) component scores were assigned using dietary data collected from the National Cancer Institute Automated Self-Administered 24-Hour Recall Tool (2014 version with 1-2 days of recalls per participant). Generalized linear regression models and two-sample t-tests were used to estimate differences in HEI-2010 component scores by food security status.

Results: Food security was significantly associated with increased dairy intake compared to food insecurity $(\beta=$ $1.0 ; \mathrm{p}=0.05$ ), and mean whole grain intake (mean \pm SD) was significantly higher in food secure compared to food insecure Indiana SNAP-Ed eligible adult participants $(1.0 \pm 2.9 ; \mathrm{p}=0.02)$.

Conclusions and Implications: These study results highlight a need to increase dairy and whole grain consumption in the SNAP-Ed eligible population, especially those experiencing food insecurity.

Funding: AgSEEd Crossroads, Purdue Health and Human Sciences Cooperative Extension Nutrition Education Program

\section{P80 Development of a Healthy Weight Management SNAP-Ed Nutrition Education Curriculum: Food Talk: Better U}

Sarah Stotz, MS, $R D, C D E$, University of Georgia;

Laurel Sanville, MS, RD; Rachelle Acitelli Reed, PhD;

Whitni McConnell, MS, RD; Mona F. Habibi, PhD;

Jung Sun Lee, PhD, RD, leejs@uga.edu, University of Georgia, 280 Dawson Hall; Department of Foods and Nutrition, Athens, GA 30602

Objective: To develop a culturally tailored, evidencebased obesity prevention program to address high burden of obesity among SNAP-Ed participants.

Description: The theory was social cognitive theory and expanded health belief model. Food Talk: Better U (FTBU) is a series of four 90-minute sessions taught by paraprofessionals focusing on improving healthy weight management practices. Lesson topics include: portion control, setting SMART goals, limiting added sugar intake, making small healthy shifts in everyday food choices, and problem-solving strategies for mitigating common barriers to healthful eating. Each lesson includes a sharing session, didactic lesson, physical activity (PA), cooking demonstration/tasting, and goal setting. Featured recipes focus on healthful versions of traditional Southern dishes. PA includes resistance exercises with emphasis on proper mechanics. Participants receive a take-home workbook for food and PA journaling, goal setting, additional physical activities, and nutrition education/PA extenders to enhance healthful practices at home (e.g., measuring cups, resistance bands).

Evaluation: A rigorous needs assessment including literature review and interviews with SNAP-Ed eligible participants and peer nutrition educators was completed prior to FTBU development. Formative evaluation was completed in a pilot county, including class observations, participant focus group, and educator interviews. Conclusions and Implications: The development of FTBU is based on systematic, comprehensive needs assessment and rigorous evaluation methods, as aligned with the new direction of SNAP-Ed guidance. FTBU will serve as a foundation for obesity prevention intervention for SNAP-Ed programming in Georgia and will be expanded to eLearning and social marketing nutrition education interventions.

Funding: Supplemental Nutrition Assistance Program Education

\section{P81 EFNEP's Families Eating Smart and Moving More: Integration of Video, Social Media, and PSE in EFNEP Delivery}

Lorelei Jones, MEd, lorelei_jones@ncsu.edu, NC State University, 516 Brickhaven Drive, Raleigh, NC 27695; Carolyn Dunn, $P h D, R D$, NC State University; Emily Foley, MS; Mary Burghardt, MS, LDN, RD, NC Department of Health and Human Services; Lori Rhew, MS, CHES; Suzanne van Rijn, MAEEd, NC State University; Josephine Cialone, MS, RD, NC Department of Health and Human Services; Kathy Andersen, $M S, R D$;

Sheree Vodika, MA, LDN, RD, NC Alliance of YMCAs

Objective: To revise a comprehensive curriculum using current evidence in nutrition, physical activity, behavior change, and adult education that integrates participant and partner engagement strategies to meet the national priorities of EFNEP. Target Audience is EFNEP eligible adult audiences with an emphasis on parents and other adult caregivers who have primary responsibility for feeding children in their care and pregnant/lactating women.

Theory, Prior Research, Rationale: The curriculum is based on Stages of Change and Social Cognitive Theory (SCT) within the framework of the Community Nutrition Education (CNE) Logic Model. The curriculum is designed with a focus on individual, family, or household level change with partner engagement strategies for policy and environmental change informed by the CNE Logic Model.

Continued on page S55 
P81 (continued)

Description: EFNEP's Families Eating Smart and Moving More is an evidence-based curriculum first developed in 2006 and revised once every five years to integrate current research as well as implementer, partner, and participant feedback. This revision includes video and social media strategies to engage participants and contemporary strategies to engage program partners in policy and environmental changes (PSE) that support healthy eating, access to safe, healthy foods, and physical activity of program participants. Partnership strategies are included in each lesson as a guide for educators.

Evaluation: Curriculum effectiveness has been measured using required pre and post 24-hour participant recall and food behavior surveys. Anecdotal success stories are collected to report policy and environmental changes.

Conclusions and Implications: Quantitative data demonstrates consistently high impacts in individual dietary change, physical activity, food resource management, and food safety for each revision of the curriculum. Evaluation of strategies from this revision will be completed over the next five years.

Funding: Expanded Food and Nutrition Education Program

\section{P82 Exploring the Role of Fathers in the Women, Infants, and Children (WIC) Supplemental Nutrition Program}

Dan Dychtwald, MS, ddychtwald@me.com, Drexel University, 1601 Cherry Street, Philadelphia, PA 19102; Brandy-Joe Milliron, PhD, Drexel University

Objective: Evidence suggests that men can play an influential role in improving pregnancy, breastfeeding, and early childhood outcomes. However, the role that programs like the Women, Infants, and Children (WIC) nutrition program play in fostering paternal participation is not known. While men may not be qualified for WIC, their partners and children may benefit from WIC's nutrition subsidies, health screenings, and nutrition education. Therefore, the primary objective of this research was to explore the experiences, expectations and attitudes of men towards WIC, including their exposure to WICEducation.

Study Design, Setting, Participants: This study employed a convergent mixed methods design combining surveys and in-depth interviews. Couples were recruited at WIC offices in Philadelphia, Pennsylvania.

Outcome Measures and Analysis: Interviews were audio-recoded and transcribed verbatim. Data were analyzed and common themes identified via thematic analysis and verified using NVivo-10 Software.

Results: Although this study is ongoing, thus far nine couples have been interviewed (mean age: 32 years; mean number of children: 2). Fathers' experiences were grouped into two primary themes. Emergent themes highlighted the lack of paternal participation, which was exemplified by $78 \%$ of men reporting no interaction with WIC (including WIC-Ed). Related sub-themes included coercion fears and WIC office inaccessibility as deterrents to paternal involvement. The second major theme, the need for paternal participation, documented the desires of fathers to improve parenting skills and their knowledge of nutrition in support of their partners and children.

Conclusions and Implications: There may be missed opportunities for fostering the male role within WIC programming, particularly WIC-Ed.

Funding: None

\section{P83 Food Security and Food Resource Management Outcomes of Haitian EFNEP Participants}

Jennifer Walsh, PhD, RD, walsh5jr@jmu.edu, James Madison University, 235 Martin Luther King Jr. Way, MSC 4301, Harrisonburg, VA 22807;

Danielle De Vries-Navarro, MS, LDN, RDN, University of Florida; Karla Shelnutt, $P h D, R D$

Objective: To determine whether there is a difference in program outcomes related to improved food security and food resource management (FRM) skills between Expanded Food and Nutrition Education Program (EFNEP) Haitian and non-Haitian participants. Target audience is Haitian and non-Haitian female EFNEP Participants in Palm Beach County, Florida.

Theory, Prior Research, Rationale: The Eating Smart, Being Active curriculum is based on Social Cognitive Theory, with published efficacy, and used in Florida's EFNEP program. Since 2010, the population of Haitian residents in south Florida has increased by over 22,000, increasing Haitian EFNEP participants. Assessment of Haitian EFNEP participants' knowledge and behavior related to program objectives is needed to identify educational opportunities.

Description: A retrospective analysis of pre- and postprogram evaluation outcomes was conducted among Haitian $(n=102)$ and non-Haitian adult EFNEP participants $(n=136)$ completing the program between October 2014 and September 2015. Trained, multilingual nutrition educators implemented the 8-week program.

Evaluation: Likert-type EFNEP checklist items, ranging from "never $=1$ " to "almost always $=5$ ", were used to assess food security (1 item) and FRM (3 items). Differences in change from pre- to post- between Haitian and non-Haitian participants were determined using Student's t-test. Food security scores increased more from pre- to post- for Haitian versus non-Haitian participants ( $1.4 \pm 1.9$ versus $0.6 \pm 1.6, \mathrm{p}=.001)$. Similarly, scores for FRM improved more for Haitian versus nonHaitian participants $(<.05)$.

Conclusions and Implications: Participants from Haiti may benefit from EFNEP in ways that enhance food security and FRM. Adapting programming to be 